\title{
Novel Type of References for Weight Aligned for Onset of Puberty - Using the Qeps Growth Model
}

Kerstin Albertsson-Wikland ( $\nabla$ kerstin.albertsson.wikland@gu.se )

University of Gothenburg

Aimon Niklasson

University of Gothenburg

Lars Gelander

University of Gothenburg

Anton Holmgren

University of Gothenburg

Andreas Nierop

University of Gothenburg

\section{Research Article}

Keywords: pubertal growth, personalized growth, biological age, SDS, weight status, longitudinal BMIselection, reference population

Posted Date: June 1st, 2021

DOI: https://doi.org/10.21203/rs.3.rs-429731/v2

License: (c) (i) This work is licensed under a Creative Commons Attribution 4.0 International License.

Read Full License

Version of Record: A version of this preprint was published at BMC Pediatrics on November 13th, 2021. See the published version at https://doi.org/10.1186/s12887-021-02954-z. 
1 Novel type of references for weight aligned for onset of puberty - using the QEPS

2 growth model

3 Kerstin Albertsson-Wikland ${ }^{1 *}$, Aimon Niklasson ${ }^{2}$, Lars Gelander ${ }^{1}$, Anton Holmgren ${ }^{2,3}$,

4 Andreas F.M. Nierop ${ }^{1,4}$

$5{ }^{1}$ Department of Physiology/Endocrinology, Institute of Neuroscience and Physiology,

6 Sahlgrenska Academy, University of Gothenburg, Gothenburg, Sweden

$7 \quad{ }^{2}$ Göteborg Pediatric Growth Research Center, Department of Pediatrics, Institute of Clinical

8 Sciences, Sahlgrenska Academy, University of Gothenburg, Sweden

$9 \quad{ }^{3}$ Department of Pediatrics, Halmstad Hospital, Halmstad, Sweden

$10{ }^{4}$ Muvara bv, Multivariate Analysis of Research Data, Leiderdorp, The Netherland,

Correspondence: Kerstin Albertsson-Wikland, MD, PhD, Professor; Department of Physiology/Endocrinology, Institute of Neuroscience and Physiology, Sahlgrenska Academy,

13 University of Gothenburg, SE 40530 Gothenburg,

Sweden; Kerstin.albertsson.wikland@gu.se Phone: +46 708828990

Short title: Pubertal weight reference

Orchid numbers: https://orcid.org/

18 KAW: 0000-0003-3167-0667; email Kerstin.albertsson.wikland@ gu.se

AN: 0000-00001-5839-4207; email Aimon@bahnhof.se; aimon.niklasson@gu.se

LG: email Lars.gelander@vgregion.se; lars.gelander@gu.se

AH: 0000-0003-0940-8726; email Anton.holmgren@ @regionhalland.se; anton.holmgren@gu.se AFMN: 0000-0002-6709-4836; nierop@muvara.nl; andreas.nierop@gu.se

Keywords: pubertal growth, personalized growth, biological age, SDS, weight status, 
Abstract

Background: Growth references are traditionally constructed relative to chronological age, despite inter-individual variations in pubertal timing. A new type of height reference was recently developed allowing growth to be aligned based on onset of pubertal growth. We here aim to develop a corresponding reference for pubertal weight.

Methods: To model QEPS-weight, 3595 subjects (1779 girls) from GrowUp ${ }_{1974}$ Gothenburg and GrowU $\mathrm{p}_{1990}$ Gothenburg were used. The QEPS-height-model was transformed to a corresponding QEPS-weight-model; thereafter, QEPS-weight was modified by an individual, constitutional weight-height-factor. Longitudinal weight and length/height measurements from 1418 individuals (698 girls) from GrowU $\mathrm{p}_{1990}$ Gothenburg were then used to create weight references aligned for height at pubertal onset (the age at 5\% of P-function growth, AgeP5). GrowUp ${ }_{1974}$ Gothenburg subgroups based on pubertal timing, stature at pubertal onset, and childhood body composition were assessed using the references.

Results: References (median, SDS) for total weight (QEPS-functions), weight specific to puberty (P-function), and weight gain in the absence of specific pubertal growth (basic weight, QES-functions), allowing alignment of individual growth based on age at pubertal onset. For both sexes, basic weight was greater than average for late maturing, tall and highBMI subgroups. The P-function-related weight was greater than average in short and lower than average in tall children, in those with high BMI, and in girls but not boys with low BMI.

Conclusions: New pubertal weight references allow individual variations in pubertal timing to be taken into consideration when evaluating growth. When used together with the comparable pubertal height reference, this will improve growth monitoring in clinical practice for identifying abnormal growth, and serve as a valuable research tool providing insight into human growth. 


\section{Background}

The growth curve of an individual child reflects both the physical and psychosocial wellbeing of that child. According to the World Health Organization (WHO), every child has the right to grow and be healthy, and growth references or standards are crucial tools for monitoring such growth (1). It is therefore important that repeated height and weight measurements are obtained for every child within a healthcare setting, and plotted alongside established growth references, preferably using a computerized system (1-3). In order to be optimal, references should be constructed using data from children growing optimally. Height references should be updated regularly in countries where the population is still experiencing a secular trend for height to increase over time, as was recently done in Sweden (4). Similarly, in some countries, updates to weight references should consider that weight development may be unhealthy in children and adolescents owing to the ongoing obesity epidemic. This issue was addressed in the newly updated references for weight in Sweden by omitting individuals with extreme obesity from the longitudinal BMI data set used to develop the tool (5).

Growth references, both for height and weight, are traditionally presented in relation to chronological age. This remains the case despite the understanding that there is a broad variation in biological age between individuals, especially during puberty $(3,6)$. As a result of the reliance on chronological age, these references are of limited use for detecting abnormal growth during adolescence. Since the year 2000, Swedish healthcare systems have used references that incorporate an estimate of ongoing, basic, childhood growth based on the childhood growth function from the ICP-growth model (7-10). Use of this growth function has proved helpful in distinguishing 'healthy' late maturers from individuals with growth failure owing to an underlying condition or disease.' When developing the most recent Swedish height references using a cohort of children born in 1990, this type of 'prepubertal' 
height reference showing ongoing basic growth was produced using the QE-functions of the QEPS-model $(11,12)$. The QEPS-model describes individual growth using four mathematical functions: a Q (Quadratic) and E (Exponential) arising before birth and resulting in the basic growth, to which a specific pubertal growth function, $\mathrm{P}$, is added; growth is ended by an $\mathrm{S}$ (stop) function (11). We were recently able to develop and publish a completely new type of height reference describing growth resulting from the specific pubertal growth function of the QEPS model. This reference allows the height of the individual to be aligned relative to the onset of pubertal growth, and provides information on total growth, as well as separating growth that is a specific result of puberty from ongoing, basic growth that is continuing during the pubertal years $(13,14)$. As changes in height and weight should ideally be evaluated simultaneously, there remains a need for a corresponding reference for both prepubertal and pubertal weight.

The aim of this study was to use the QEPS-model to develop new references for weight during adolescence taking biological maturation into account. The weight references will be constructed using data from a homogeneous, longitudinally followed population of healthy children born at term to non-smoking mothers and Nordic parents, selected from the GrowUp $p_{1990}$ Gothenburg cohort born in Sweden in around 1990 (5). It will be possible to use the weight references to evaluate pubertal growth in a child, irrespective of the chronological age at which pubertal growth begins in that individual. Separate references will be available for (i) total weight (modeled by the Q, E, P and S functions), (ii) weight gain that is specific to puberty (by the $\mathrm{P}$ function), and (iii) weight gain in the absence of growth specific to puberty (basic weight growth) (by the Q, E and S functions). We will also explore the usefulness of the new references for monitoring weight by analyzing subgroups of children 
from the GrowUp p $_{1974}$ Gothenburg cohort categorized according to the timing of puberty (early, average, late), height at start of puberty (tall, short) and body composition during childhood (high body mass index (BMI), low BMI), as used for exploring the comparable pubertal height reference (14).

\section{Methods}

\section{Materials}

\section{Subset selection from GrowUp $1974 \& 1990$ Gothenburg cohorts for modelling QEPS weight}

Data were retrieved from two community based observational growth studies conducted in high schools in Gothenburg, Sweden: GrowUp ${ }_{1974}$ Gothenburg and GrowUp ${ }_{1990}$ Gothenburg $(9,15,16)$. Individuals with extreme BMI values relative to the GrowUp ${ }_{1974}$ Gothenburg

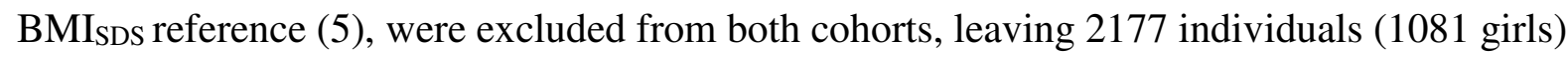

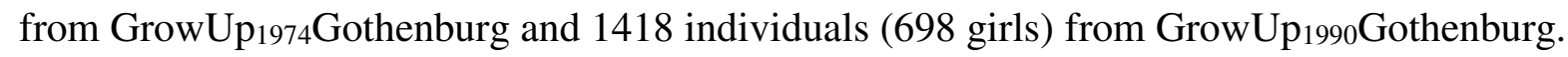
The latter cohort served as the reference population for the recently published LMS weight, weight-for-height, and BMI references (5). Both cohorts were combined to form a group of 3595 subjects (1779 girls) which was used to construct the QEPS weight model needed for developing the references.

\section{Reference population from GrowUp1990Gothenburg cohort}

The QEPS weight reference population included 1418 individuals (698 girls) from the GrowUp ${ }_{1990}$ Gothenburg cohort; this was the same cohort used to create the previously published LMS total weight, weight-for-height, and BMI references (see Supplemental Table 
128 S1 of(5)). Children in this cohort were healthy (see Table 1 of(5)), born between 1989 and 1991 at full term (gestational age (GA) 37-43 weeks) in Sweden to Nordic parents with nonsmoking mothers, and had information available on longitudinal growth until adult height. For more information see Albertsson-Wikland et al $(5,13)$.

\section{Subgroups in the GrowUp 1974 Gothenburg cohort used for exploring the novel} reference

Data from subgroups of healthy children from the GrowUp ${ }_{1974}$ Gothenburg cohort (2177 subjects; 1081 girls) were used in order to explore the utility of the new pubertal weight reference as a research tool. These investigations looked in turn at the impact of grouping children according to age at onset of puberty, height at onset of puberty and childhood BMI. According to pubertal age: early $(<-1.5 \mathrm{yrs})$, average $( \pm 0.25 \mathrm{yrs})$ or late $(>+1.5 \mathrm{yrs})$ onset of puberty (see Supplemental Table S3 of(14)); when age at TPHV (PHV from the total height growth curve) was used to estimate pubertal age. According to height: tall (>+1.5SDS) or short (<-1.5SDS) stature at onset of puberty. According to childhood BMI: high BMI $(>+1.5 \mathrm{SDS})$ or low BMI $(<-1.5 \mathrm{SDS})$.

\section{QEPS weight method}

The QEPS height model (11), was first transformed to a corresponding QEPS weight model.

147 For detailed information see Supplemental for QEPS-weight model, including Supplemental

148 Figure S1, S2, S3. QEPS weight was thereafter modified by an individual constitutional

149 factor, a so-called weight-height-factor $(W H F)$ : WHF=0 stands for a 'normal body

150 constitution', $W H F>0$ for a heavier than normal and $W H F<0$ for a leaner than normal body 
constitution. Traditional references for total and prepubertal weight according to

152 chronological age were computed in two steps comparable to the 'QEPS method used for the references' section in Supplement of (13), but with QEPS height functions replaced by corresponding QEPS weight functions fitted on observed individual weight data, referred to as the QEPS weight method.

We compared the total weight references obtained using the QEPS weight method with the previously published weight reference obtained by applying the LMS method (5). Both references were generated using data from the same population, Supplemental Figure S4. The two mean reference curves were similar; however, variance was smaller for the QEPSderived compared with the LMS-derived reference became smaller, since it was based on fitted weight functions, excluding all sorts of weight error, whereas the LMS-derived reference was fitted on the observed weight measurements, including such error.

Thereafter, a new type of reference was generated for pubertal weight designed so that growth was aligned according to onset of puberty for the individual, identified based on height-specific $P$-function growth, defined as AgeP5, when 5\% of height Pmax was obtained (Supplemental Figure S2 (14)) (12). All longitudinal QEPS weight functions for individuals in the reference population were aligned according to height AgeP5. For more information see Supplement. 


\section{Results}

\section{Total and prepubertal/basic weight references vs chronological age}

177 Figure 1 shows the novel weight reference in $\mathrm{kg}$ (left axis) and in $\mathrm{kg}^{0.5}$ (right axis) according reference) shown in black.

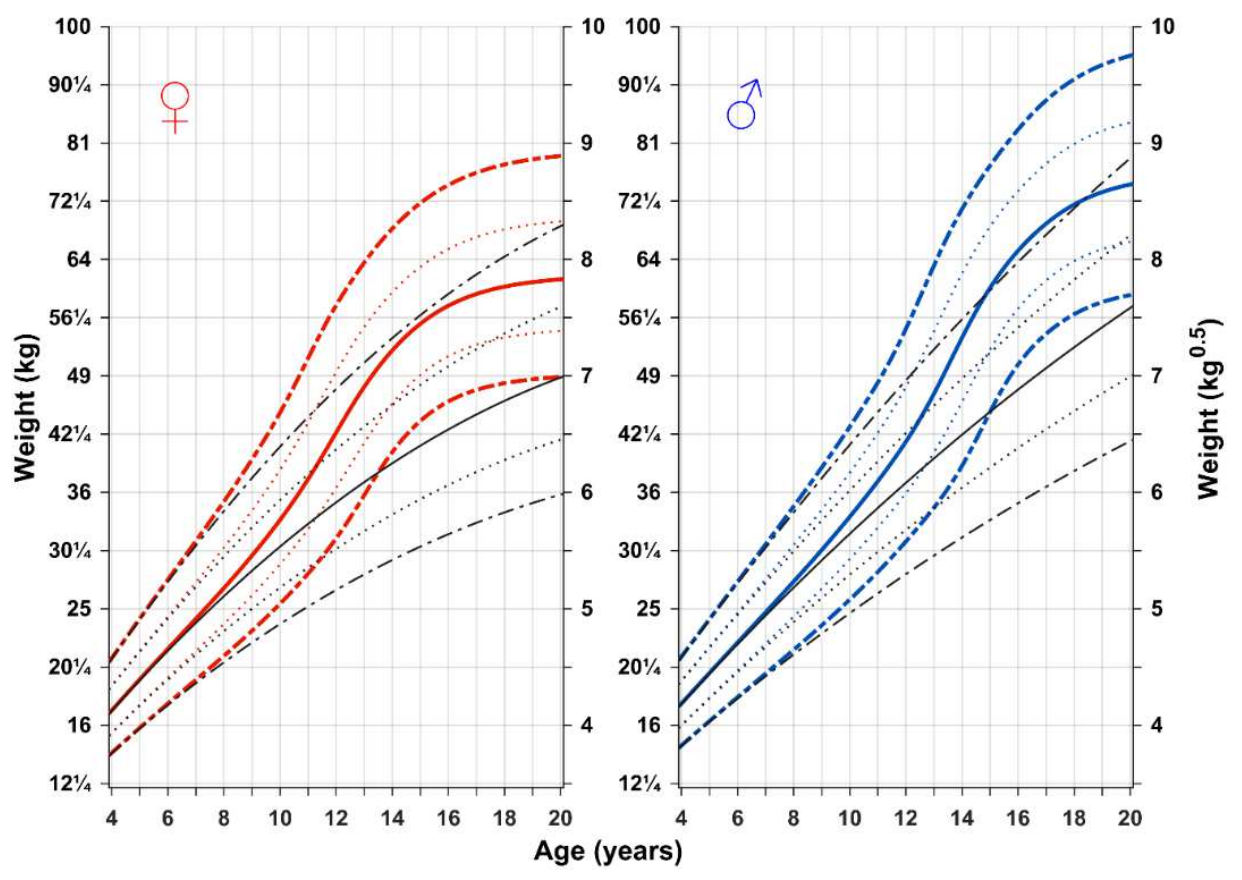

Legend Figure 1: Chronological age reference for total and basic growth. References for weight (total weight and weight gained independently of puberty (basic growth $=$ QESfunction)) for girls (left) and boys (right) aged 4-20 years. Mean total weight (red/blue solid line, $\pm 1 \mathrm{SDS}$ (red/blue dotted line), and $\pm 2 \mathrm{SDS}$ (red/blue dashed line) and mean basic weight (black). 
191 Figure 2 shows the reference for total (QEPS) and basic (QES-function) weight in kg (left axis) and $\mathrm{kg}^{0.5}$ (right axis) for girls and boys aligned according to the onset of puberty, estimated as AgeP5. In Figure 2 lower panel, specific P-function growth has been included alongside the curves shown in Figure 2 upper panel. To capture changes in weight relative to the acceleration in height that occurs during puberty, weight references in both figures are depicted from 4 years before to 10 years after the onset of the pubertal growth spurt.
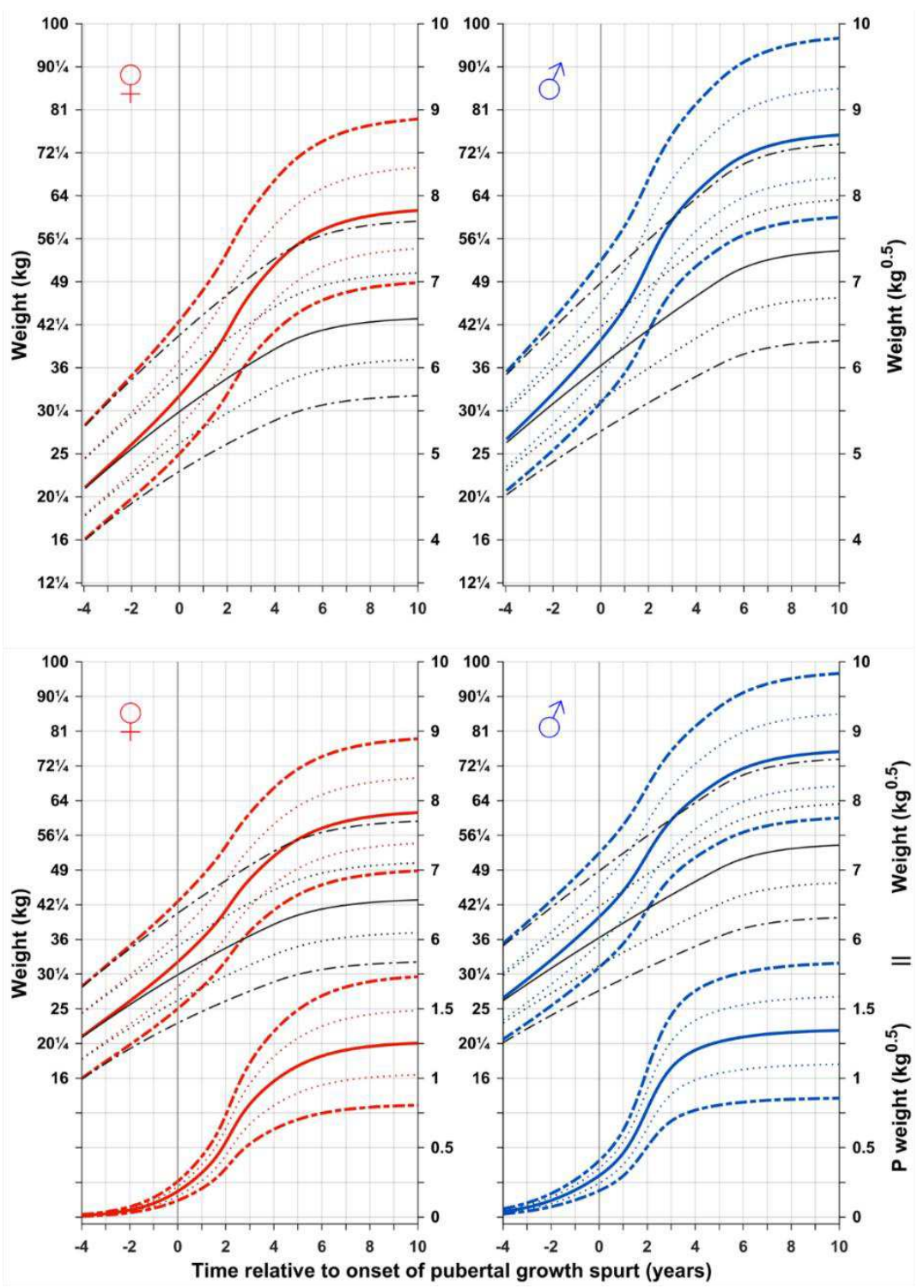
200 Legend Figure 2 upper panel: References in $\mathrm{kg}$ (left axis) and in $\mathrm{kg}^{0.5}$ (right axis) for total

201

202

203

204

205

206

207

208

209

210

211

212

213

214

215

216

217

weight and the prepubertal/basic weight (weight gained independently of puberty (QESfunction) for girls (red, left) and boys (blue, right). Curves are aligned for age at onset of pubertal growth spurt. Mean total weight (red/blue solid line, \pm 1 SDS (red/blue dotted line), and \pm 2 SDS (red/blue dashed line) and mean basic weight (black). Figure 2 lower panel: References shown in Figure 2 upper panel with the addition of a puberty-specific weight reference showing weight gain resulting from the P-function of the QEPS (P weight). Specific $P$-function-derived weight as mean, \pm 1 SDS (dotted line), and \pm 2 SDS (dashed line). The individual onset of puberty was identified and aligned based on the age at which 5\% (AgeP5) of the total specific P-function growth (Pmax) had occurred.

\section{Rational for a puberty-aligned weight reference}

Figure 3 shows total weight relative to chronological age for subgroups of girls and boys with an early, average, and late onset of puberty. As expected, weight gain occurred sooner than average in children with an early onset of puberty, and later than average in children with a late onset of puberty. Thus, high lightening the inadequate usefulness for many adolescents of an ordinary chronological age weight reference due to the broad variation in chronological age at pubertal maturation within a population. 

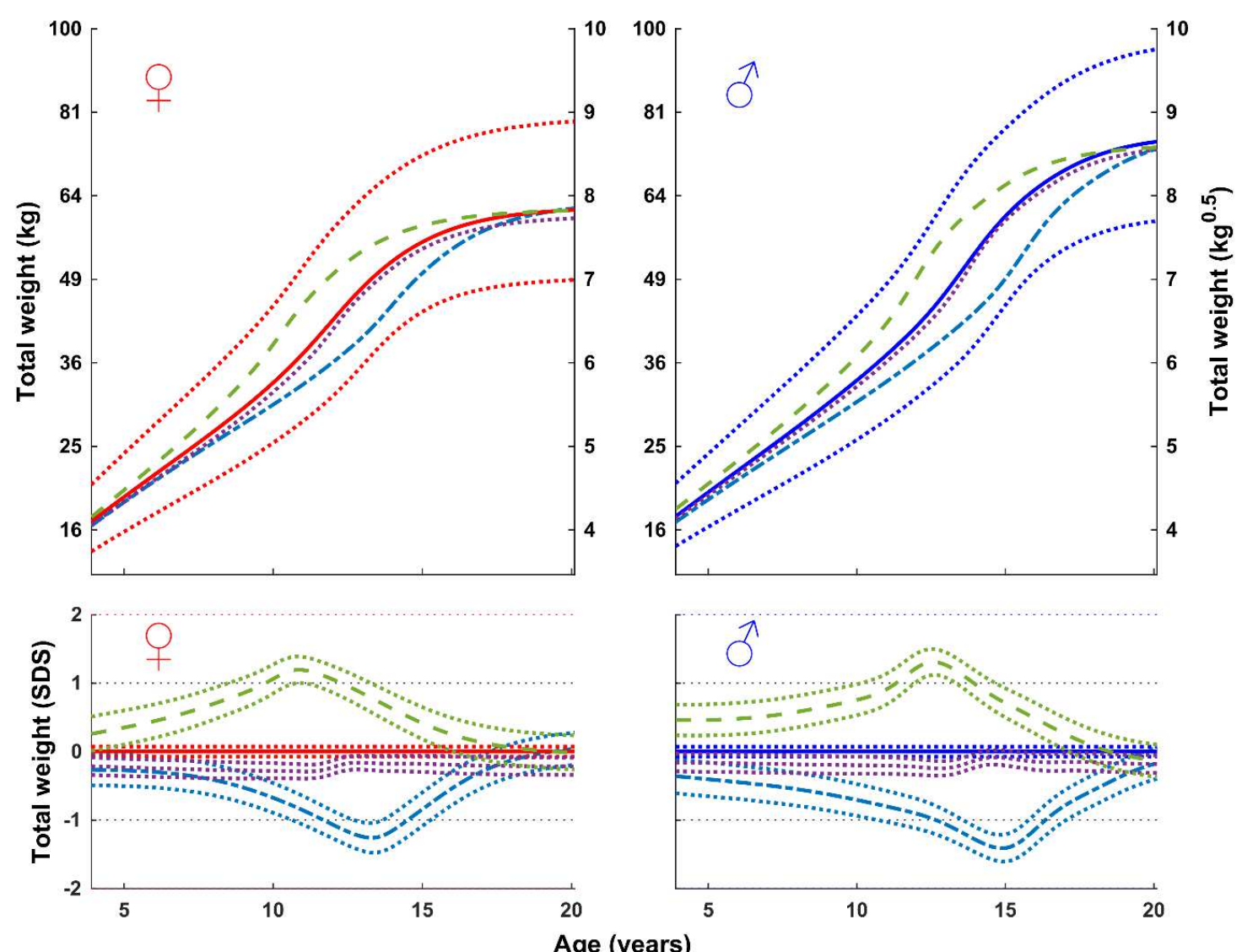

Age (years)

219

220

221

222

223

224

225

226

227

Legend Figure 3: Mean total weight (in $\mathrm{kg}$, top; in SDS with 95\% CI, bottom) according to chronological age for girls (left) and boys (right) from the GrowUp ${ }_{1974}$ Gothenburg cohort grouped according to onset of puberty (early, $<-1.5 \mathrm{yrs}(--)$ ), average, $\pm 0.25 \mathrm{yrs}(\bullet \bullet)$, and late, $>+1.5 y r s(--)$ ). Data are visualized alongside the reference for total mean weight (thick solid lines) and \pm 2 SDS (dotted red (left) and blue (right) lines).

\section{Exploring pubertal weight in subgroups from the GrowU p $_{1974}$ Gothenburg cohort}

Timing of puberty: Figure 4 shows weight (total (QEPS), basic (QES) and pubertal (Pfunction)) relative to the onset of puberty for subgroups of girls and boys with an early, average, and late onset of puberty. The basic component of weight gain was lower than average in the early puberty group and higher than average in the late puberty group. Specific weight gain during puberty was similar for all three groups. Total weight gain was similar in all groups 

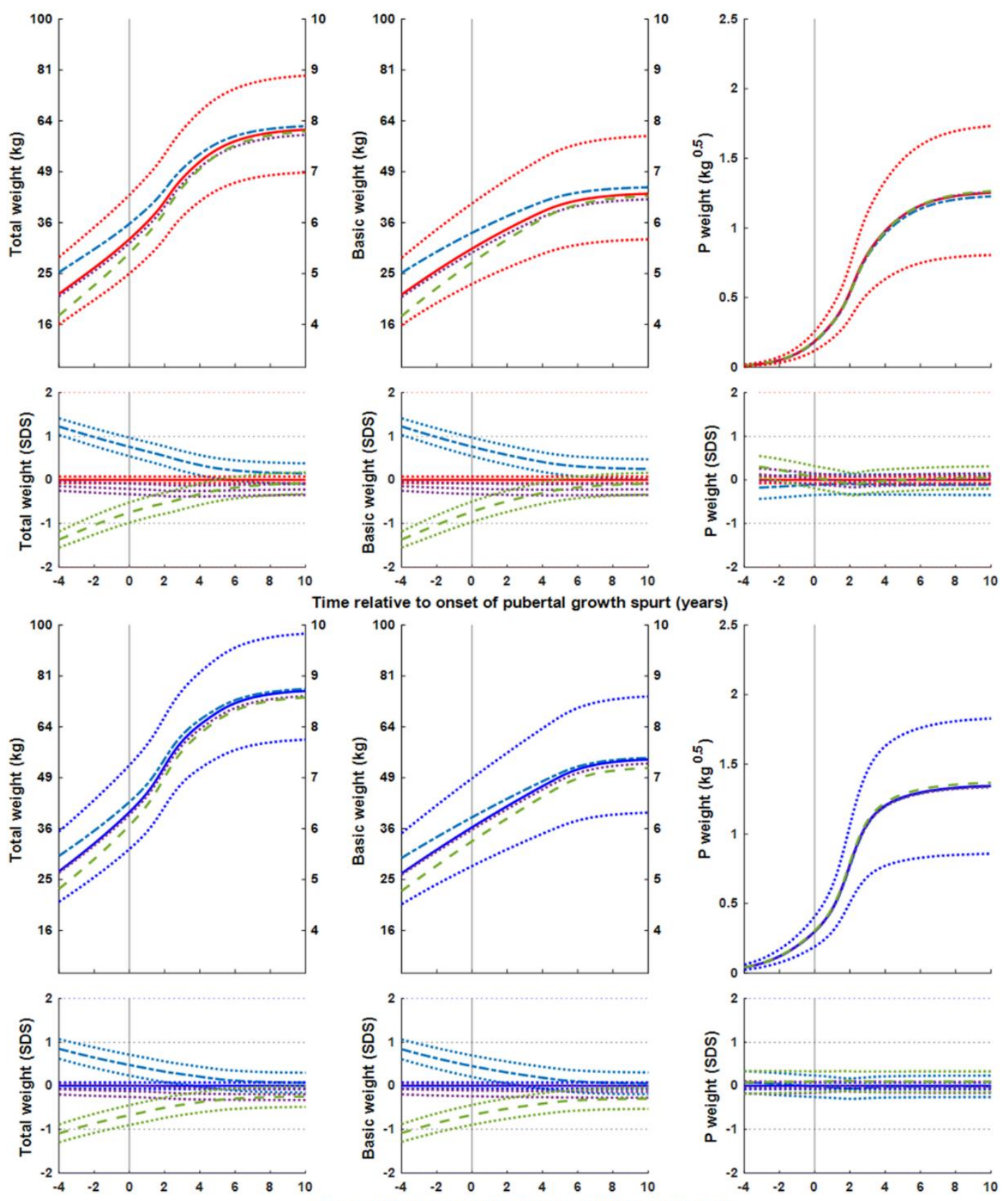

Legend Figure 4. Mean total, basic and puberty-specific weight gain (in kg, top; in SDS with 95\% CI, bottom) relative to onset of puberty for girls (red at the top) and boys (blue at the bottom) grouped according to onset of puberty (early, $<-1.5$ yrs (- - ) , average, \pm 0.25 yrs ( $\bullet$ $\bullet$ ), and late, $>+1.5 y$ rs $\left(--_{-}\right)$). Mean total weight (left panels), basic weight growth (middle panels), and specific pubertal weight growth (right panels) from the GrowU $\mathrm{p}_{1974}$ Gothenburg cohort. Curves are aligned for age at onset of pubertal growth spurt. Data are visualized alongside the new reference for total mean weight (thick blue solid lines) and \pm 2 SDS (dotted blue lines). 
242 Stature: Figure 5 shows weight (total (QEPS), basic (QES) and pubertal (P-function)) for

243 girls and boys according to height at the onset of puberty. The basic component of weight

244 gain was higher than average in girls and boys classed as tall at the onset of puberty, and

245 lower than average in girls and boys classed as short. In contrast, specific weight gain during puberty was lower than average for the tall group and higher than average for the short group.
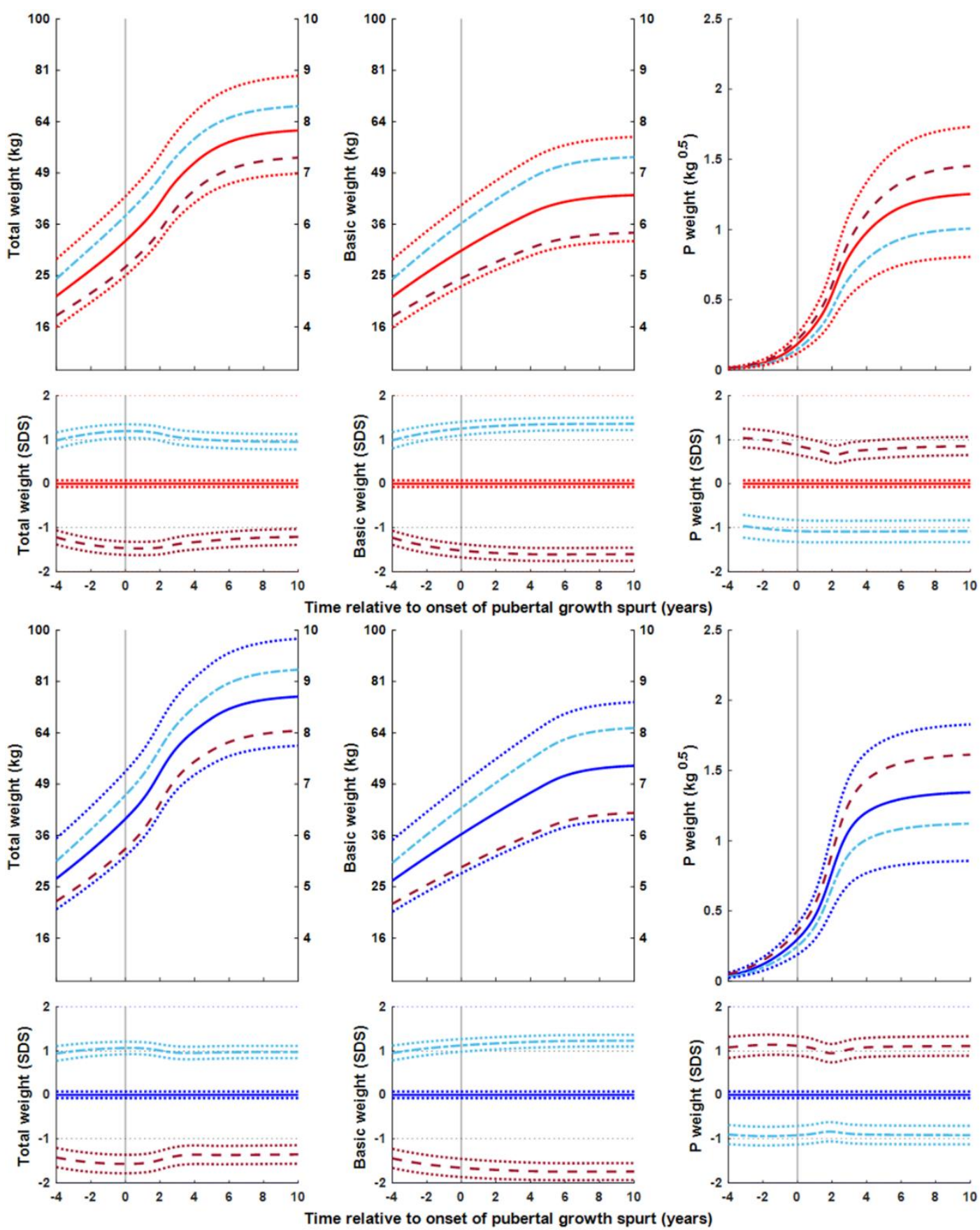
248 Legend Figure 5: Mean total, basic and puberty-specific weight gain (in kg, top; in SDS with $95 \% \mathrm{CI}$, bottom) relative to the onset of puberty for girls (red at the top) and boys (blue at the bottom) according to height at the onset of puberty (tall, >+1.5 SDS (- - -) and short, $<-1.5$ SDS (- - )). Mean total weight (left panels), basic weight growth (middle panels), and specific pubertal weight growth (right panels) from the GrowUp ${ }_{1974}$ Gothenburg cohort.

253 Curves are aligned for age at onset of pubertal growth spurt. Data are visualized alongside the new reference for total mean weight (thick red solid lines) and \pm 2 SDS (dotted red lines).

Body Size: Figure 6 shows weight (total (QEPS), basic (QES) and pubertal (P-function)) for

257 girls and boys according to BMI during childhood. The basic component of weight gain was higher than average in the high BMI group and lower than average in the low BMI group. Puberty-specific weight gain differed between the sexes: weight gain specific to the pubertal function was slightly lower than average in both the high and low BMI groups for girls, but in only the high BMI group for boys. Specific pubertal weight gain for boys in the low BMI group was similar to the average for the whole population. 

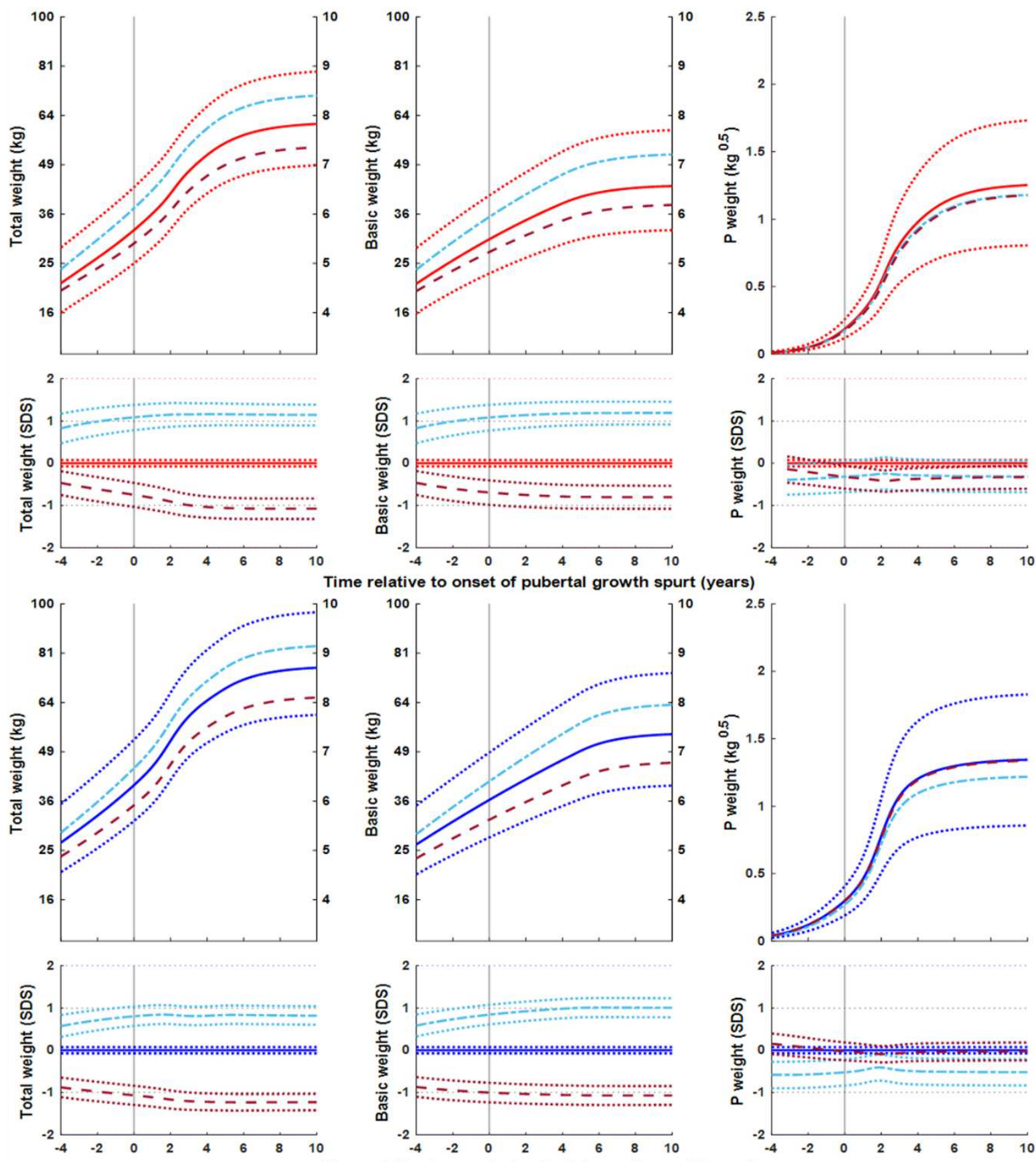

264 Legend Figure 6: Total, basic and puberty-specific weight gain (in kg, top; in SDS with 95\% CI, bottom) relative to the onset of puberty for girls (red at the top) and boys (blue at the bottom) according to BMI in childhood (high BMI, >+1.5SDS (- - -) and low BMI, $<-1.5 \operatorname{SDS}(---))$. Mean total weight (left panels), basic growth (middle panels), and specific pubertal growth (right panels) in girls and boys from the GrowU $\mathrm{p}_{1974}$ Gothenburg cohort. Curves are aligned for age at onset of pubertal growth spurt. Data are visualized alongside the new reference for total mean weight (thick red solid lines) and \pm 2 SDS. 
272 A manual procedure can be undertaken in order to monitor pubertal growth prospectively for an individual child using the new pubertal-age-growth charts developed from the pubertalage-adjusted reference. A growth chart with both total and prepubertal height (13), as well as the present weight references, should be used for easy identification of the 'height-take off' at the start of puberty (13). Figure 7 describes how to use the pubertal-age-adjusted reference

277 prospectively to assess total weight.
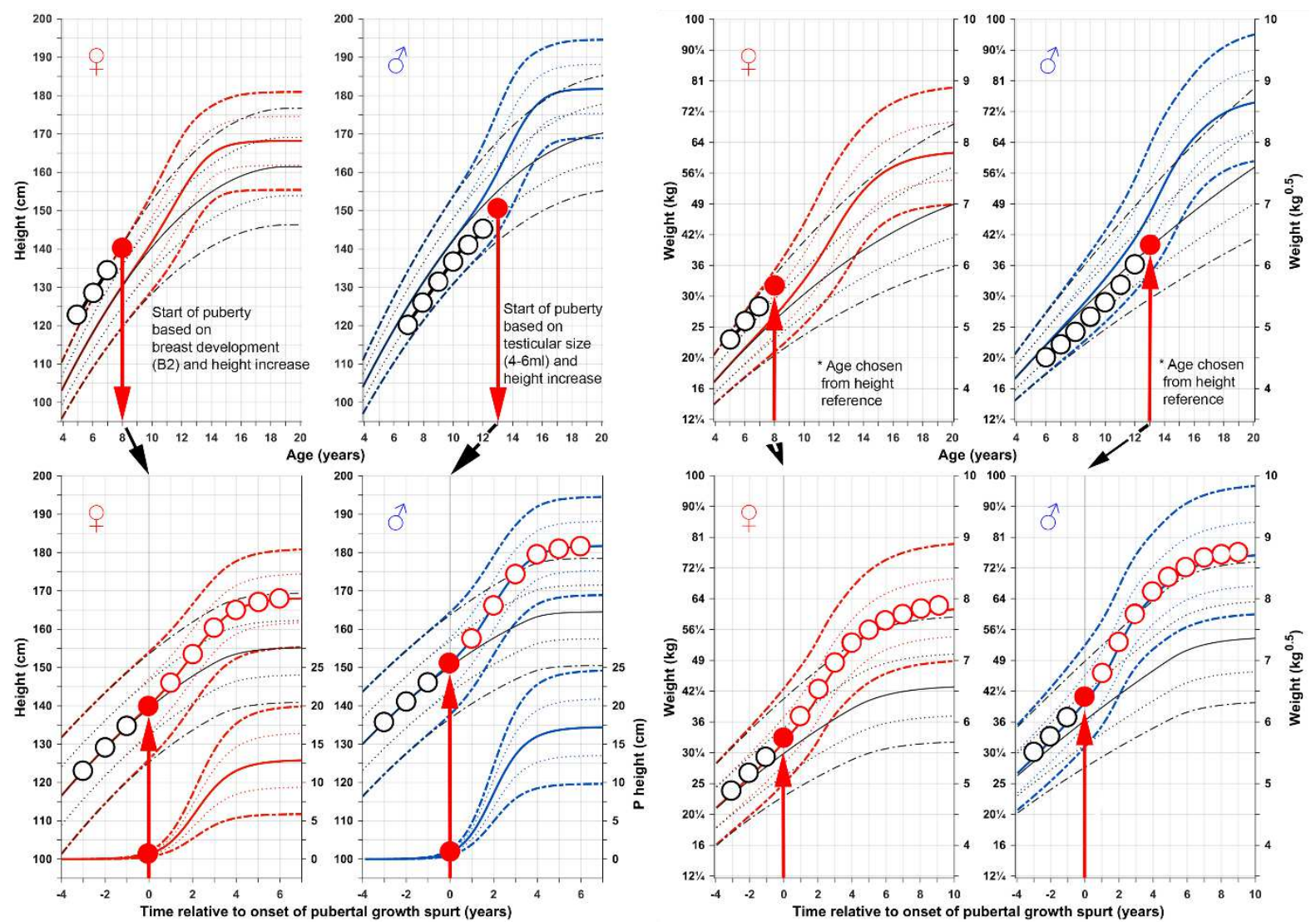

Legend Figure 7: A guide for prospective use of the pubertal-age-adjusted reference for total weight $(\mathrm{kg}$ and $\sqrt{\mathrm{kg}})$ and height $(\mathrm{cm})$ for girls $(+)$ and boys $\left({ }^{\Uparrow}\right)$.

Individual age adjustment is made only from the height measurement at the chronological age (C-age) at which puberty started (see upper left traditional C-age-reference for girls and boys) (14), by using the height increase from the individual prepubertal growth curve (as drawn in upper left panel) through the individual measuring points. This occurs when secondary sex 
characteristics develop; early breast development stage, B2 in girls (red, upper left panel), or testicular volume increase to 4-6 $\mathrm{ml}$ in boys (blue, upper left panel)..

Height and weight according to $\mathrm{C}$-age and $\mathrm{P}$-age, ie the age adjusted to reflect the start of puberty are shown for an 8-year-old girl and a 13-year-old boy (14). Height (cm) and weight (in $\mathrm{kg}$ or $\sqrt{\mathrm{kg}}$ ) according to $\mathrm{C}$-age at the onset of puberty, and to P-age, after adjustment for age at onset of the pubertal growth spurt are depicted as red dots (all panels), based only on age in the height references for each sex respectively. Corresponding heights and weights are then moved to puberty-adjusted age $=$ zero, in the P-age-references shown in the lower panels (left panels for height and right for weight). Thereafter, all measured heights and weights are depicted at ages/times recalculated in relation to the specific onset of puberty in that individual, labelled on the $\mathrm{x}$-axis as 'Time from onset of growth spurt (years)'. Thus, changes in weight and height in the years preceding the pubertal growth spurt can be evaluated using the novel reference. Note, the weight increase precedes the height increase in relation to puberty.

\section{Discussion}

\section{New prepubertal and pubertal weight references}

We present the first pubertal weight references to be developed that can be adjusted to reflect the biological maturation of the individual child. The new references include median values and SDS that allow alignment of individual weight data based on the time/age at onset of the pubertal growth spurt. These references were created using the growth functions of the QEPS-model which have previously been successfully applied to create a height reference that can be aligned for the onset of pubertal growth in the individual (14). By enabling the clinician to simultaneously adjust both weight and height based on the maturational stage of the individual, these two references promise to improve the precision of the evaluation of growth during adolescence (14). 
As well as enabling weight to be assessed relative to maturational stage, the weight references generated allow us to look separately at the prepubertal and pubertal components of weight gain. By separating weight gain related specifically to puberty (P-function) from other weight gain (QE-function), it is possible to look at ongoing, basic weight gain during adolescence that is unrelated to puberty. Thus, these references will form a novel tool with which to investigate both puberty-independent and puberty-dependent changes in weight.

\section{Weight changes precede height changes}

By virtue of the alignment of weight data with the onset of the pubertal growth spurt, these references will allow us to look in more detail at the changes in weight that are known to precede the increase in height associated with the onset of puberty. Changes in weight before the acceleration of growth during puberty were already shown by Backman almost a century ago (17). It is well established that pubertal growth begins with characteristic sex-specific changes in body fat mass, that occur earlier in girls than boys, and this suggests a link between energy storage in adipose tissue and pubertal maturation (18). The new references will be new tool separating weight in relation to pubertal onset and by that increase our knowledge about changes in weight before the increase in height associated with puberty (Supplemental Figure S3).

\section{Usefulness of a reference separating the growth functions during puberty}

The benefit of using a growth model is not only to describe growth but also to be able to analyse how different growth functions can be related to the mechanisms underlying the regulation of growth $(19,20)$. Here, thanks to the mathematical growth functions from the 
QEPS-weight and the QEPS-height models, we present a tool for investigating weight gain in new ways and with higher precision than previously possible. In this analysis, we explored differences in weight gain between groups of children with different characteristics prior to entering puberty. A novel finding from this analysis was that basic weight gain was greater in boys and girls who were late maturers or tall at the start of puberty, when compared to either the reference, or those of average pubertal timing or stature. The contrary, with lower than average basic weight gain, was found for those who underwent early pubertal maturation or had short stature. P-function related weight gain was greater than average in short individuals and lower than average in tall individuals. Previously, we investigated children with different BMI, and found a positive relationship between BMI and greater than average gains in basic height and lower than average gains in puberty-specific height (21). Results from the present study extend these findings, showing that a high BMI is also associated with greater than in girls, and average in boys.

\section{A QEPS-weight model behind puberty-aligned weight references}

Developing references for weight that are aligned for individual onset of puberty, require a growth model for individual weight. As only a model for weight in infancy and early childhood was available (22-25), we had to develop a model for the entire growth period that was good enough for this purpose. We also had to deal with the problem that weight measurements are known to be subject to greater daily variations than are expected for measurements of height. We therefore took the approach of transforming height values into 
corresponding values for weight in order to develop the weight model. Thus, the QEPSweight model was supported by a transformation of fitted QEPS-height functions (11), into corresponding QEPS-weight functions. For better fitting results, introduction of only one individual factor was necessary; a specific individual weight parameter $(W H F)$ in the weight model that was not predicted by height. However, the QEPS-weight model could also have been constructed and fitted directly on observed weight measurements using the six individual weight parameters, while optimizing the basic four shape-invariant QEPS-weight functions. In our experience, it would have resulted in considerable overfitting of the weight measurements, because of higher standard error of the fit for weight than for height. For height, the residual error was mostly owing to measurement error and time of day, whereas for weight, the residual variation was caused by many factors and could not simply be explained. Residual variation around individual predicted weight for normal body constitution might, for instance, also be related to individual variation in the relationship of fat /muscle distributions as estimated by body compartment analyses (DEXA) or subcutaneous fat measures, waist circumference or other comparable measurements. There are models for human growth and body dynamics (26). Here, we have tried to find a good enough QEPS-weight model using the individual height information, however, other approaches or fine-tuning of the method remains to be developed.

\section{Biological correlates of individual WHF}

The introduction of an individual WHF originated from the improved fitting of the model, described above and in Supplement. This gives rise to the question as to whether there is any biological correlate /connection to this factor. Adult height of an individual can fairly accurate be predicted by growth during infancy (ie attained height at 2 years of age), and 
delayed timing of transition from infancy to childhood growth corresponds to reduced adult height. Thus, it seems that there is a critical window in early life for changes in growth (19). Moreover, clinically, there also appears to be a critical window during infancy for changing body size (correlate to WHF); it is known to be extremely difficult to change body size later on in life, if a child was thin (low WHF) or obese (high WHF) during infancy (27). There is increasing evidence of a role for epigenetic programming during prenatal and early postnatal periods in determining child growth and development and later development of diseases. For instance, prenatal epigenetics have been shown to change IGF-I expression in several ways (28-30), and the Dutch Famine showed that famine during early and mid-gestation affects metabolism later in life, including increased risk for obesity(31). There is also increasing evidence that variations in early postnatal nutrition have epigenetic effects on developmental programming and may result in the development of cardiovascular diseases, overweight, obesity, diabetes, and other chronic conditions (32-35).

\section{Prospective monitoring of growth in individual children using the new references and}

\section{future perspectives}

These new weight references will allow the creation of updated computerized references for use in healthcare settings. Until computerized versions of the puberty-aligned references for height and weight are available, a manual procedure can be undertaken in order to monitor pubertal growth prospectively for an individual child using the new references (see Figure 7). A growth chart with both total and prepubertal height (13), as well as the present weight references, should be used for easy identification of the 'height-take off' at the start of puberty(13). The use of these new references will help to determine whether or not we should be concerned about the growth of late-maturing children who appear to be underweight, or 
early-maturing children who appear to be overweight, based on assessments relative to

407

408

409

410

411

412

413

414

415

416

417

418

419

420

421

422

423

424

425

426

427

chronological age. The availability of this type of reference is an important step towards more meaningful and informative monitoring of weight development of an individual during the adolescent years. The next step will be to develop references for other growth-related parameters, such as BMI.

\section{Conclusion}

We here present new types of weight references both for prepubertal weight in relation to chronological age, and for weight during pubertal years aligned for the onset of the pubertal growth spurt in the individual. When used together with the prepubertal and pubertal height references $(13,14)$, the new weight references will allow for improved growth monitoring during adolescence for detecting abnormal growth. The new references will also serve as valuable research tools that will help to provide new insights into human growth.

\section{Declarations:}

Ethical approval: Ethical approval was obtained from the Regional Ethics Review Board in Gothenburg, (Ad 91-92/131 -93 and Ad 444-08 T062-09). Informed consent was given by participants and parents of individuals $<18$ years of age. Studies were conducted in accordance with the principles of the Declaration of Helsinki.

\section{Consent of Publications: Not Applicable}


Availability of data and materials: the generated and analyzed during the current study are not publicly available but are available from the corresponding author on reasonable request

Competing interest: AFMN works for Muvara, Multivariate Analysis of Research Data, competing interests.

Research funding: The authors acknowledge financial support from the Governmental grants under the ALF agreement, ALFGBG-719041, ALFGBG-812951, the Region Halland Grants, and the Foundation Växthuset for children.

Author contributions: KAW is the principal investigator of the study populations used.

AFMN performed the modeling work for the QEPS model described here and made the QEPS function estimations, reference values, and Figures 1-6 \& Supplemental Figures S1-S4. AN made Figure 7 and statistics. KAW, AN, AH, LG, and AFMN have all made a substantial contribution to the conception, design, analysis, and interpretation of these data. KAW wrote the first draft and AFMN the methods part. All authors revised the manuscript critically for intellectual content and approved the final version to be submitted for publication.

Acknowledgements: The authors are grateful for the contributions of the students of the $12^{\text {th }}$ grade 1998/9 from the Gothenburg area schools, and their school nurses; the study team led by C Pfeiffer-Mosesson and A Olsson; for Data support given by L Ljungberg and B Bengtsson and for careful editing and language revision by $\mathrm{H}$ Crofts. 
$453 \quad 1 . \quad$ Who Multicentre Growth Reference Study G, de Onis M. WHO Child Growth

454 Standards based on length/height, weight and age. Acta Pædiatrica. 2006; 95:76-85

455

456

457

458

2. Sankilampi U, Saari A, Laine T, Miettinen PJ, Dunkel L. Use of electronic health records for automated screening of growth disorders in primary care. JAMA. 2013; 310:1071-2

$3 . \quad$ Cole TJ. The development of growth references and growth charts. Annals of human biology. 2012; 39:382-94

4. Holmgren A, Niklasson A, Aronson AS, Sjoberg A, Lissner L, Albertsson-Wikland K. Nordic populations are still getting taller - secular changes in height from the 20th to 21st century. Acta Paediatr. 2019; 108:1311-20

5. Albertsson-Wikland K, Niklasson A, Gelander L, Holmgren A, Sjoberg A, Aronson AS, et al. Swedish references for weight, weight-for-height and body mass index: The GrowUp 1990 Gothenburg study. Acta Paediatr. 2021; 110:537-48

6. Tanner JM, Whitehouse RH, Takaishi M. Standards from birth to maturity for height, weight, height velocity, and weight velocity: British children, 1965. I. Archives of disease in childhood. 1966; 41:454-71

$4687 . \quad$ Karlberg J. On the modelling of human growth. Statistics in medicine. 1987; 6:185-92

469 8. Karlberg J, Fryer JG, Engstrom I, Karlberg P. Analysis of linear growth using a

470 mathematical model. II. From 3 to 21 years of age. Acta paediatrica Scandinavica. 1987; 337:12-29

$4719 . \quad$ Albertsson-Wikland K, Luo ZC, Niklasson A, Karlberg J. Swedish population-based

472 longitudinal reference values from birth to 18 years of age for height, weight and head

473 circumference. Acta Paediatr. 2002; 91:739-54

$474 \quad 10 . \quad$ Gelander L, Hagenäs L, Albertsson-Wikland K. Tillväxtkurvan i skolhälsovården. Tillväxt och kroppslig mognad 6-18 år. Manual och Lilla tillväxtskolan. Stockholm: Kommentus; 2003 11. Nierop AF, Niklasson A, Holmgren A, Gelander L, Rosberg S, Albertsson-Wikland K. Modelling individual longitudinal human growth from fetal to adult life - QEPS I. J Theor Biol. 2016; 406:143-65

12. Holmgren A, Niklasson A, Gelander L, Aronson AS, Nierop AFM, Albertsson-Wikland K. Insight into human pubertal growth by applying the QEPS growth model. BMC pediatrics. 2017; $17: 107$

13. Albertsson-Wikland K, Niklasson A, Holmgren A, Gelander L, Nierop AFM. A new Swedish reference for total and prepubertal height. Acta Paediatr. 2020; 109:754-63

14. Albertsson-Wikland KG, Niklasson A, Holmgren A, Gelander L, Nierop AFM. A new type of pubertal height reference based on growth aligned for onset of pubertal growth. J Pediatr Endocrinol Metab. 2020; 33:1173-82

15. Sjöberg A, Barrenäs ML, Brann E, Chaplin JE, Dahlgren J, Mårild S, et al. Body size and lifestyle in an urban population entering adulthood: the 'Grow up Gothenburg' Study. Acta Paediatr. 2012; 101:964-72

16. Holmgren A, Niklasson A, Nierop AFM, Gelander L, Aronson AS, Sjoberg A, et al. Estimating secular changes in longitudinal growth patterns underlying adult height with the QEPS model: the Grow Up Gothenburg cohorts. Pediatric research. 2018; 84:41-9

17. Backman G. Gewichtswachstum des Mannes. Wilhelm Roux' Archiv für Entwicklungsmechanik der Organismen 1940; 140:285-314

18. Vizmanos B, Marti-Henneberg C. Puberty begins with a characteristic subcutaneous body fat mass in each sex. European journal of clinical nutrition. 2000; 54:203-8 19. Hochberg Z, Albertsson-Wikland K. Evo-Devo of Infantile and Childhood Growth. Pediatric research. 2008; 64:2-7 

Evolution, First Edition.: Wiley-Blackwell, Publishers; 2012 height gain is inversely related to peak BMI in childhood. Pediatric research. 2017; 81:448-54 Statistics in medicine. 2007; 26:2547-64 23. Beath KJ. Infant growth modelling and assessment of Growth. In: Preedy VR, editor Handbook of growth and Growth monitoring in health and disease: Springer; 2012

24. Ahmadi S, Bodeau-Livinec F, Zoumenou R, Garcia A, Courtin D, Alao J, et al. children with repeated measurements. BMJ open. 2020; 10:e035785

25. Botton J, Scherdel P, Regnault N, Heude B, Charles MA, Group EM-CCS. Postnatal weight and height growth modeling and prediction of body mass index as a function of time for the study of growth determinants. Ann Nutr Metab. 2014; 65:156-66

26. Rahmandad H. Human growth and body weight dynamics: an integrative systems model. PLoS One. 2014; 9:e114609

27. Koletzko B, Brands B, Poston L, Godfrey K, Demmelmair H, Early Nutrition P. Early nutrition programming of long-term health. The Proceedings of the Nutrition Society. 2012; 71:371-8 28. Fu Q, McKnight RA, Yu X, Wang L, Callaway CW, Lane RH. Uteroplacental insufficiency induces site-specific changes in histone $\mathrm{H} 3$ covalent modifications and affects DNA-histone $\mathrm{H} 3$ positioning in day 0 IUGR rat liver. Physiological genomics. 2004; 20:108-16

29. Tosh DN, Fu Q, Callaway CW, McKnight RA, McMillen IC, Ross MG, et al. Epigenetics of programmed obesity: alteration in IUGR rat hepatic IGF1 mRNA expression and histone structure in rapid vs. delayed postnatal catch-up growth. Am J Physiol Gastrointest Liver Physiol. 2010; 299:G1023-9

30. Zinkhan EK, Fu Q, Wang Y, Yu X, Callaway CW, Segar JL, et al. Maternal Hyperglycemia Disrupts Histone 3 Lysine 36 Trimethylation of the IGF-1 Gene. Journal of nutrition and metabolism. 2012; 2012:930364

31. Roseboom T, de Rooij S, Painter R. The Dutch famine and its long-term consequences for adult health. Early human development. 2006; 82:485-91

32. Barker DJ, Osmond C, Kajantie E, Eriksson JG. Growth and chronic disease: findings in the Helsinki Birth Cohort. Annals of human biology. 2009; 36:445-58

33. Eriksson JG, Forsen TJ, Kajantie E, Osmond C, Barker DJ. Childhood growth and hypertension in later life. Hypertension. 2007; 49:1415-21

34. Forsdahl A. Living conditions in childhood and subsequent development of risk factors for arteriosclerotic heart disease. The cardiovascular survey in Finnmark 1974-75. Journal of epidemiology and community health. 1978; 32:34-7

35. Barker DJ, Osmond C, Forsen TJ, Kajantie E, Eriksson JG. Trajectories of growth among children who have coronary events as adults. The New England journal of medicine. 2005; 353:18029 


\section{Legends to the Figures:}

547 Legend Figure 1: Chronological age reference for total and basic growth. References for weight (total weight and weight gained independently of puberty (basic growth $=$ QESfunction)) for girls (left) and boys (right) aged 4-20 years. Mean total weight (red/blue solid line, \pm 1 SDS (red/blue dotted line), and \pm 2 SDS (red/blue dashed line) and mean basic weight (black).

Legend Figure 2 upper panel: References in $\mathrm{kg}$ (left axis) and in $\mathrm{kg}^{0.5}$ (right axis) for total weight and the prepubertal/basic weight (weight gained independently of puberty (QESfunction) for girls (red, left) and boys (blue, right). Curves are aligned for age at onset of pubertal growth spurt. Mean total weight (red/blue solid line, \pm SDS (red/blue dotted line), and \pm 2 SDS (red/blue dashed line) and mean basic weight (black). Figure 2 lower panel:

References shown in Figure 2 upper panel with the addition of a puberty-specific weight reference showing weight gain resulting from the P-function of the QEPS (P weight). Specific P-function-derived weight as mean, \pm 1 SDS (dotted line), and \pm 2 SDS (dashed line). The individual onset of puberty was identified and aligned based on the age at which 5\% (AgeP5) of the total specific P-function growth (Pmax) had occurred.

Legend Figure 3: Mean total weight (in kg, top; in SDS with 95\% CI, bottom) according to chronological age for girls (left) and boys (right) from the Grow $\mathrm{Up}_{1974}$ Gothenburg cohort grouped according to onset of puberty (early, $<-1.5$ yrs $(---)$, average, $\pm 0.25 \mathrm{yrs}(\bullet \bullet \bullet)$, and late, $>+1.5 y r s(--))$. Data are visualized alongside the reference for total mean weight (thick solid lines) and \pm 2 SDS (dotted red (left) and blue (right) lines). 
Legend Figure 4. Mean total, basic and puberty-specific weight gain (in kg, top; in SDS with 95\% CI, bottom) relative to onset of puberty for girls (red at the top) and boys (blue at the bottom) grouped according to onset of puberty (early, $<-1.5 \mathrm{yrs}(---)$, average, $\pm 0.25 \mathrm{yrs}$ $\bullet$ ), and late, $>+1.5$ yrs $(---)$ ). Mean total weight (left panels), basic weight growth (middle panels), and specific pubertal weight growth (right panels) from the GrowUp $p_{1974}$ Gothenburg cohort. Curves are aligned for age at onset of pubertal growth spurt. Data are visualized alongside the new reference for total mean weight (thick blue solid lines) and \pm 2 SDS (dotted blue lines).

Legend Figure 5: Mean total, basic and puberty-specific weight gain (in kg, top; in SDS with $95 \%$ CI, bottom) relative to the onset of puberty for girls (red at the top) and boys (blue at the bottom) according to height at the onset of puberty (tall, >+1.5 SDS (- - -) and short, $<-1.5$ SDS (- - )). Mean total weight (left panels), basic weight growth (middle panels), and specific pubertal weight growth (right panels) from the GrowUp ${ }_{1974}$ Gothenburg cohort. Curves are aligned for age at onset of pubertal growth spurt. Data are visualized alongside the new reference for total mean weight (thick red solid lines) and \pm SSDS (dotted red lines).

Legend Figure 6: Total, basic and puberty-specific weight gain (in kg, top; in SDS with 95\% CI, bottom) relative to the onset of puberty for girls (red at the top) and boys (blue at the bottom) according to BMI in childhood (high BMI, >+1.5SDS (- - -) and low BMI, $<-1.5 \operatorname{SDS}(---))$. Mean total weight (left panels), basic growth (middle panels), and specific pubertal growth (right panels) in girls and boys from the GrowUp ${ }_{1974}$ Gothenburg cohort.

Curves are aligned for age at onset of pubertal growth spurt. Data are visualized alongside the new reference for total mean weight (thick red solid lines) and \pm 2 SDS. 
593 Legend Figure 7: A guide for prospective use of the pubertal-age-adjusted reference for total

594 weight $(\mathrm{kg}$ and $\sqrt{\mathrm{kg}})$ and height $(\mathrm{cm})$ for girls $(\uparrow)$ and boys $(ð)$.

595 Individual age adjustment is made only from the height measurement at the chronological age

596 (C-age) at which puberty started (see upper left traditional C-age-reference for girls and boys)

597 (14), by using the height increase from the individual prepubertal growth curve (as drawn in

598 upper left panel) through the individual measuring points. This occurs when secondary sex

599 characteristics develop; early breast development stage, B2 in girls (red, upper left panel), or

600 testicular volume increase to $4-6 \mathrm{ml}$ in boys (blue, upper left panel)..

601 Height and weight according to C-age and P-age, ie the age adjusted to reflect the start of 602 puberty are shown for an 8-year-old girl and a 13-year-old boy (14). Height $(\mathrm{cm})$ and weight

603 (in $\mathrm{kg}$ or $\sqrt{\mathrm{kg}}$ ) according to C-age at the onset of puberty, and to P-age, after adjustment for 604 age at onset of the pubertal growth spurt are depicted as red dots (all panels), based only on 605 age in the height references for each sex respectively. Corresponding heights and weights are 606 then moved to puberty-adjusted age $=$ zero, in the P-age-references shown in the lower panels 607 (left panels for height and right for weight). Thereafter, all measured heights and weights are depicted at ages/times recalculated in relation to the specific onset of puberty in that individual, labelled on the x-axis as 'Time from onset of growth spurt (years)'. Thus, changes 610 in weight and height in the years preceding the pubertal growth spurt can be evaluated using the novel reference. Note, the weight increase precedes the height increase in relation to puberty. 
Figures

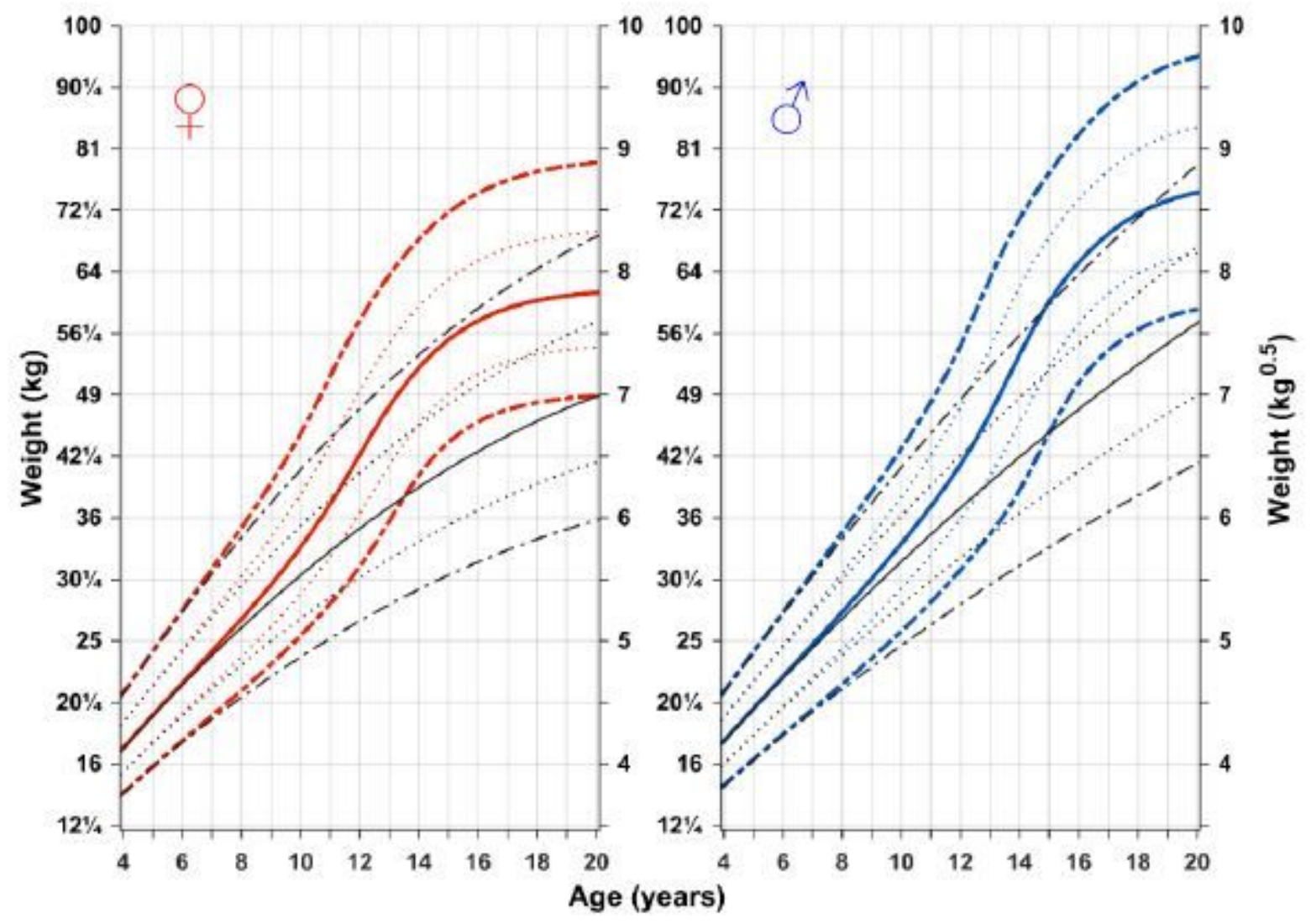

\section{Figure 1}

Chronological age reference for total and basic growth. References for weight (total weight and weight gained independently of puberty (basic growth = QES-function)) for girls (left) and boys (right) aged 4-20 years. Mean total weight (red/blue solid line, \pm 1 SDS (red/blue dotted line), and $\pm 2 S D S$ (red/blue dashed line) and mean basic weight (black). 

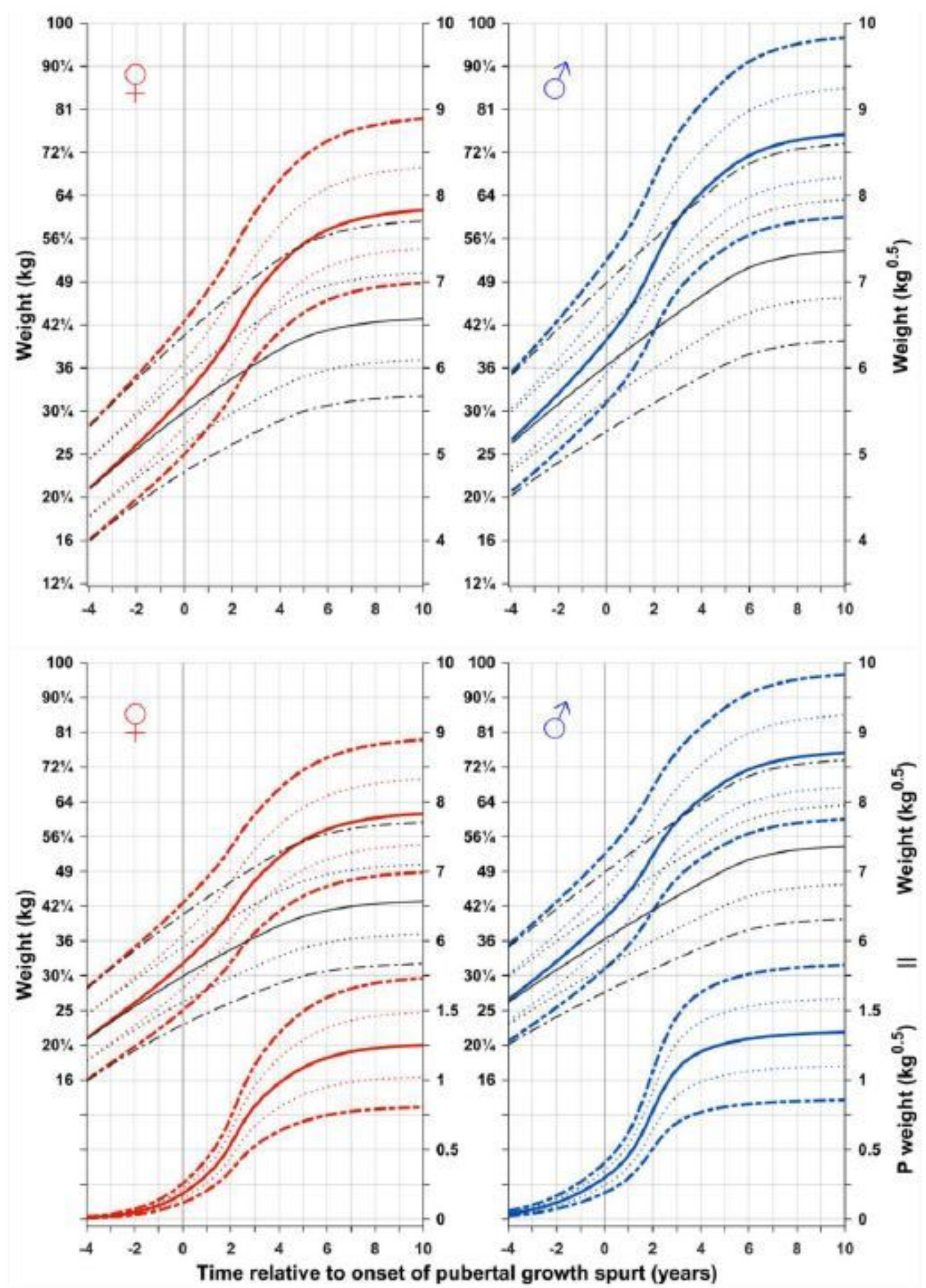

Figure 2

upper panel: References in $\mathrm{kg}$ (left axis) and in $\mathrm{kg0} 0.5$ (right axis) for total weight and the prepubertal/basic weight (weight gained independently of puberty (QES- function) for girls (red, left) and boys (blue, right). Curves are aligned for age at onset of pubertal growth spurt. Mean total weight (red/blue solid line, \pm 1 SDS (red/blue dotted line), and \pm 2 SDS (red/blue dashed line) and mean basic weight (black). Figure 2 lower panel: References shown in Figure 2 upper panel with the addition of a 
puberty-specific weight reference showing weight gain resulting from the P-function of the QEPS ( $P$ weight). Specific P-function-derived weight as mean, \pm 1 SDS (dotted line), and $\pm 2 S D S$ (dashed line). The individual onset of puberty was identified and aligned based on the age at which 5\% (AgeP5) 560 of the total specific P-function growth (Pmax) had occurred.
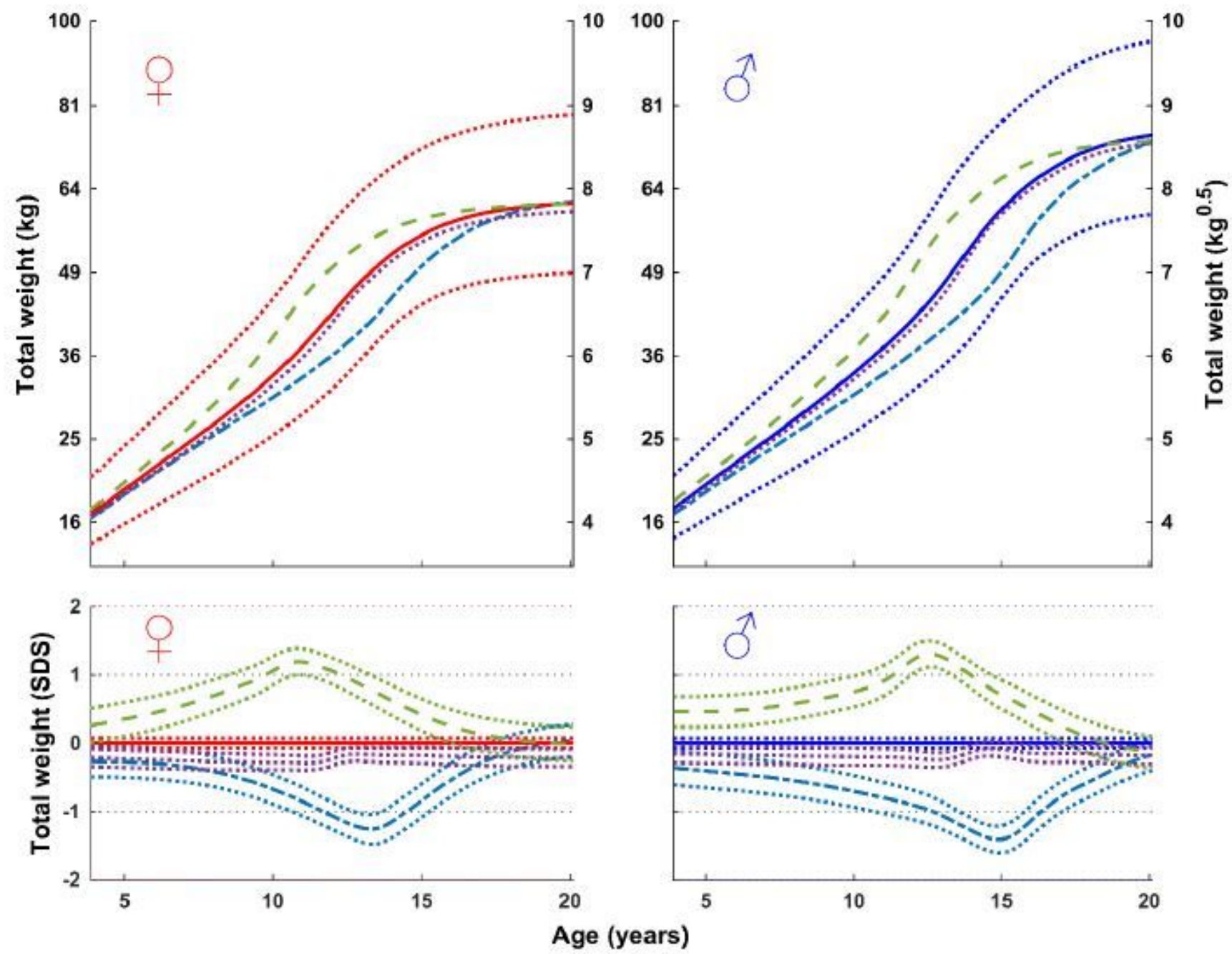

Figure 3

Mean total weight (in kg, top; in SDS with $95 \% \mathrm{Cl}$, bottom) according to chronological age for girls (left) and boys (right) from the GrowUp1974 Gothenburg cohort grouped according to onset of puberty (early, $<-1.5 y r s(--)$, average, $\pm 0.25 y r s(\cdots)$, and late, $>+1.5 y r s(--))$. Data are visualized alongside the reference for total mean weight (thick solid lines) and \pm 2 SDS (dotted red (left) and blue (right) lines). 

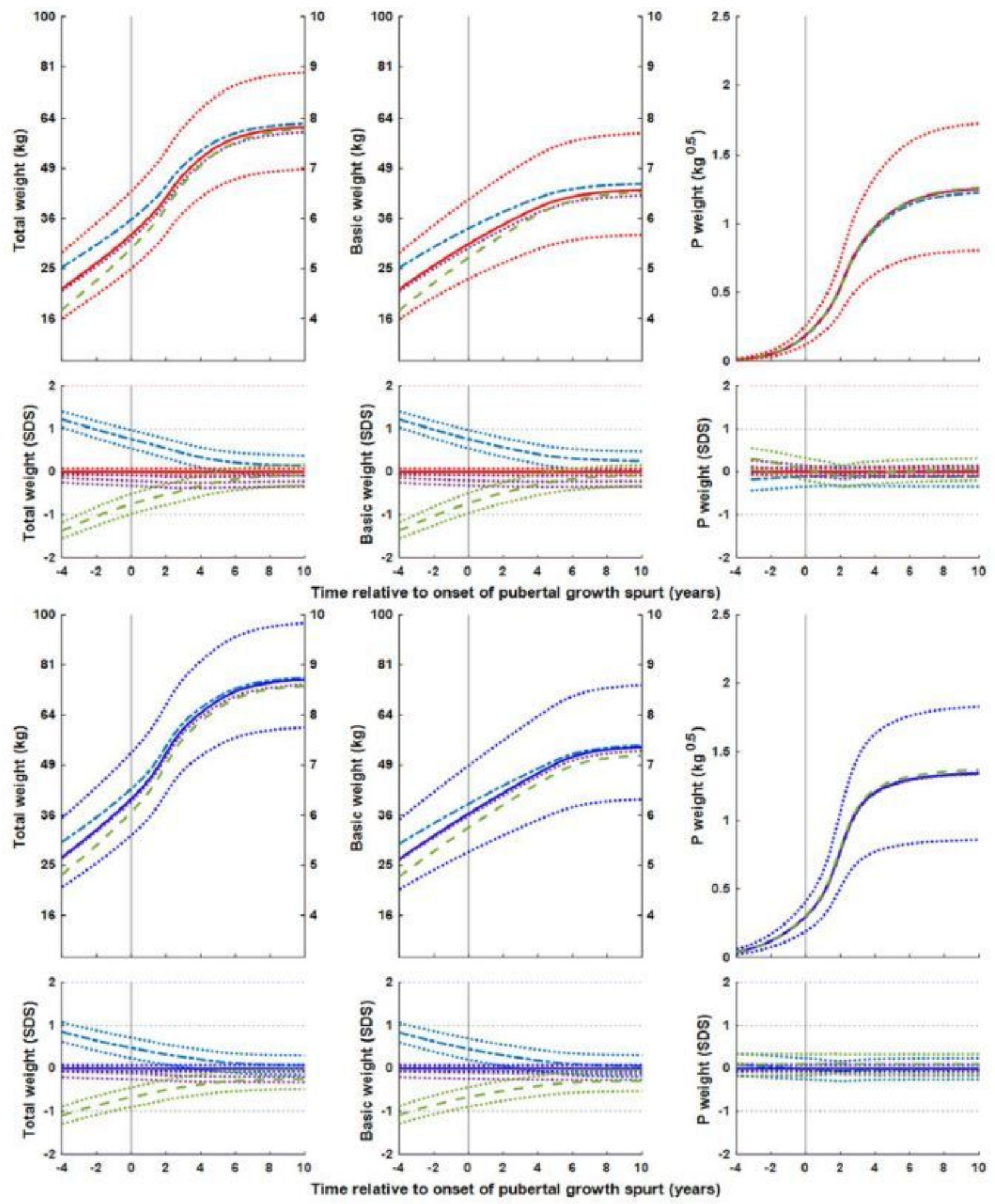

\section{Figure 4}

Mean total, basic and puberty-specific weight gain (in $\mathrm{kg}$, top; in SDS with 95\% $\mathrm{Cl}$, bottom) relative to onset of puberty for girls (red at the top) and boys (blue at the bottom) grouped according to onset of puberty (early, $<-1.5 y r s(--)$, average, $\pm 0.25 y r s(\cdots)$, and late, $>+1.5 y r s(--)$ ). Mean total weight (left panels), basic weight growth (middle panels), and specific pubertal weight growth (right panels) from the GrowUp1974Gothenburg cohort. Curves are aligned for age at onset of pubertal growth spurt. Data are 
visualized alongside the new reference for total mean weight (thick blue solid lines) and $\pm 2 S D S$ (dotted blue lines).

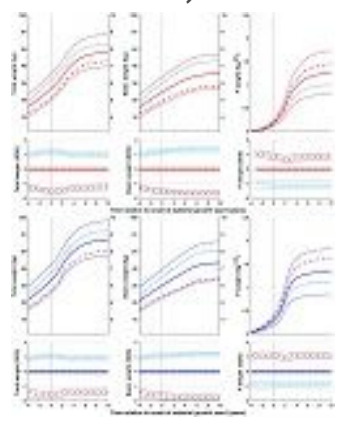

\section{Figure 5}

Mean total, basic and puberty-specific weight gain (in $\mathrm{kg}$, top; in SDS with $95 \% \mathrm{Cl}$, bottom) relative to the onset of puberty for girls (red at the top) and boys (blue at the bottom) according to height at the onset of puberty (tall, >+1.5 SDS (- - ) and short, <-1.5 SDS (-- )). Mean total weight (left panels), basic weight growth (middle panels), and specific pubertal weight growth (right panels) from the GrowUp1974Gothenburg cohort. Curves are aligned for age at onset of pubertal growth spurt. Data are visualized alongside the new reference for total mean weight (thick red solid lines) and \pm 2 SDS (dotted red lines). 

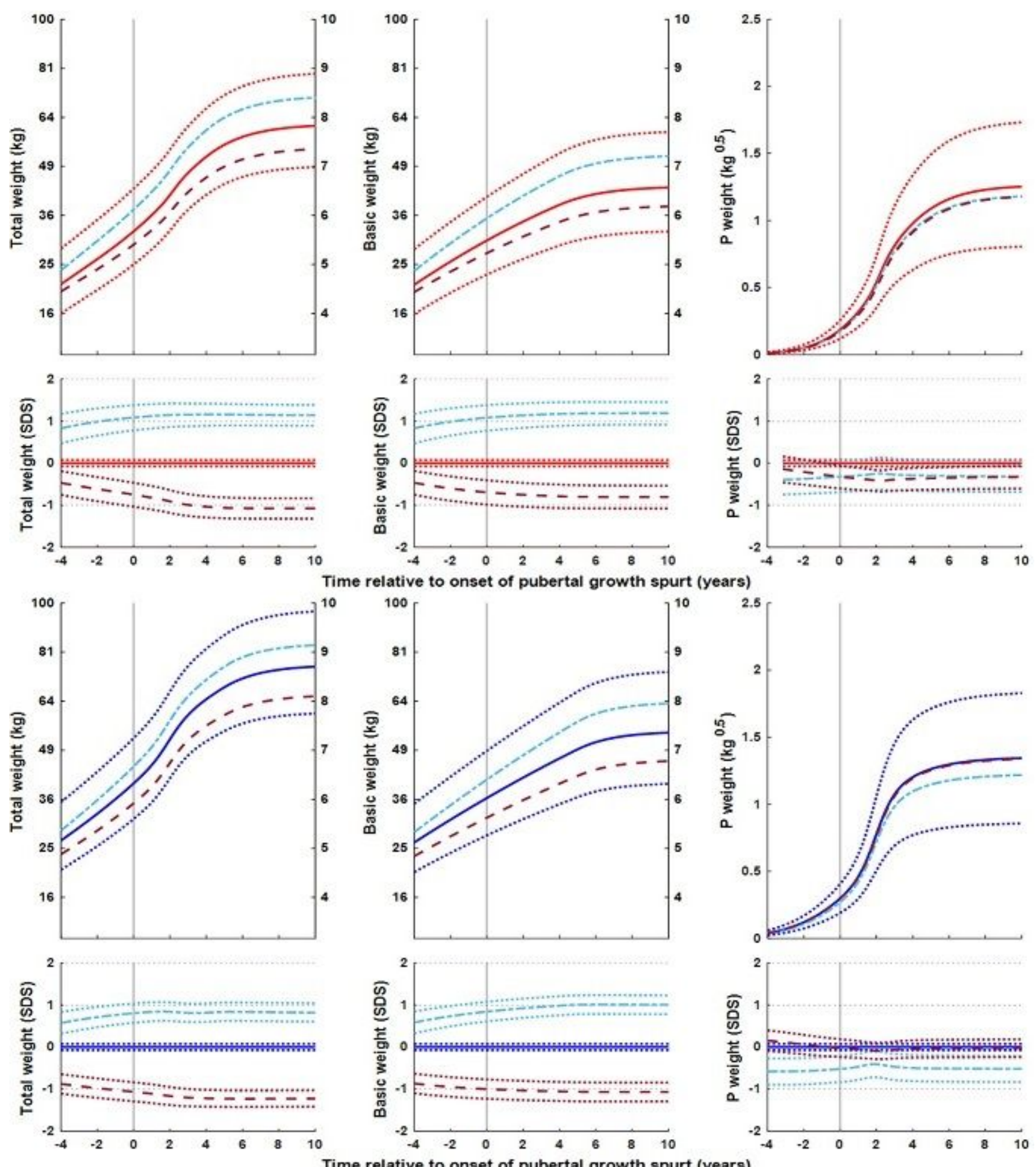

Figure 6

Total, basic and puberty-specific weight gain (in $\mathrm{kg}$, top; in SDS with $95 \% \mathrm{Cl}$, bottom) relative to the onset of puberty for girls (red at the top) and boys (blue at the bottom) according to BMI in childhood (high BMI, $>+1.5$ SDS $(---)$ and low BMI, <-1.5SDS (-- )). Mean total weight (left panels), basic growth (middle panels), and specific pubertal growth (right panels) in girls and boys from the GrowUp1974Gothenburg 
cohort. Curves are aligned for age at onset of pubertal growth spurt. Data are visualized alongside the new reference for total mean weight (thick red solid lines) and $\pm 2 S D S$.
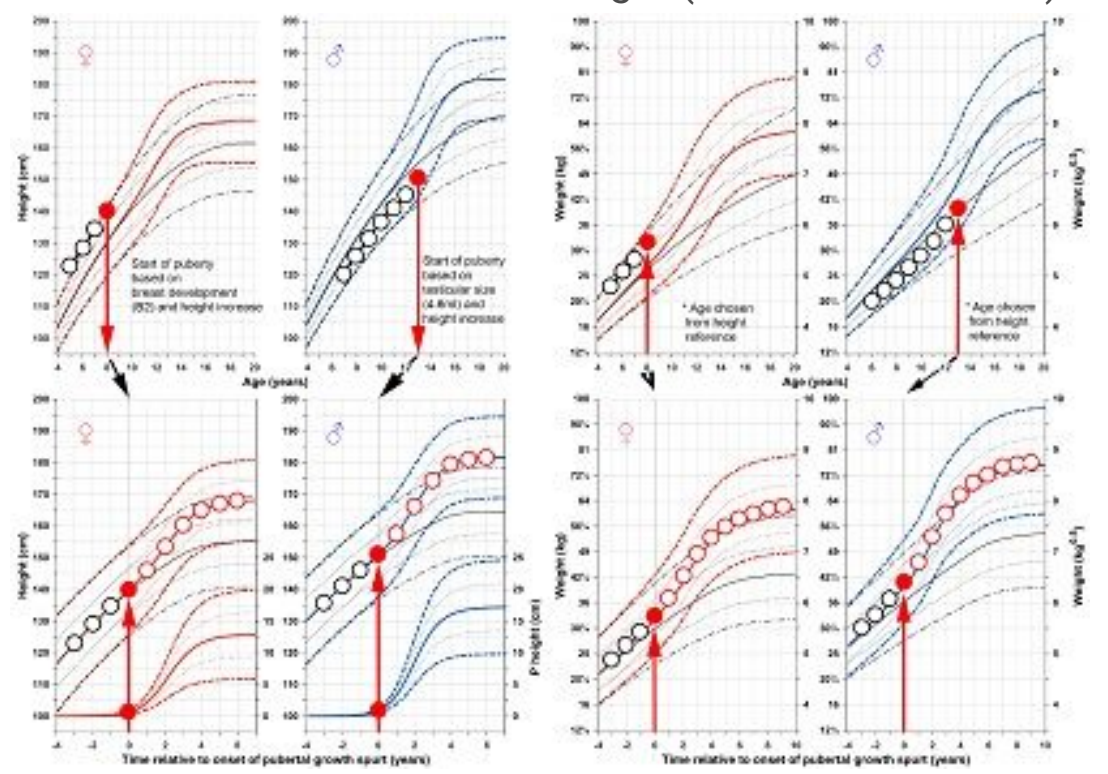

Figure 7

A guide for prospective use of the pubertal-age-adjusted reference for total weight ( $\mathrm{kg}$ and $\sqrt{\mathrm{kg}}$ ) and

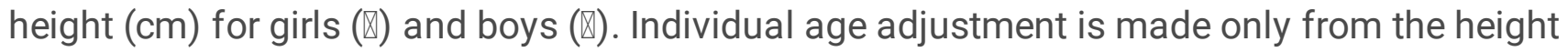
measurement at the chronological age (C-age) at which puberty started (see upper left traditional C-agereference for girls and boys) (14), by using the height increase from the individual prepubertal growth curve (as drawn in upper left panel) through the individual measuring points. This occurs when secondary sex characteristics develop; early breast development stage, B2 in girls (red, upper left panel), or testicular volume increase to $4-6 \mathrm{ml}$ in boys (blue, upper left panel).. Height and weight according to Cage and P-age, ie the age adjusted to reflect the start of puberty are shown for an 8-year-old girl and a 13year-old boy (14). Height ( $\mathrm{cm}$ ) and weight (in $\mathrm{kg}$ or $\sqrt{ } \mathrm{kg}$ ) according to $\mathrm{C}$-age at the onset of puberty, and to P-age, after adjustment for age at onset of the pubertal growth spurt are depicted as red dots (all panels), based only on age in the height references for each sex respectively. Corresponding heights and weights are then moved to puberty-adjusted age $=$ zero, in the P-age-references shown in the lower panels (left panels for height and right for weight). Thereafter, all measured heights and weights are depicted at ages/times recalculated in relation to the specific onset of puberty in that individual, labelled on the x-axis as 'Time from onset of growth spurt (years)'. Thus, changes in weight and height in the years preceding the pubertal growth spurt can be evaluated using the novel reference. Note, the weight increase precedes the height increase in relation to puberty.

\section{Supplementary Files}

This is a list of supplementary files associated with this preprint. Click to download.

- Ref420210331suppl.pdf 